\title{
Free Vibration Analysis of Functionally Graded Beams with General Elastically End Constraints by DTM
}

\author{
Nuttawit Wattanasakulpong $^{1^{*}}$, Variddhi Ungbhakorn ${ }^{2}$ \\ ${ }^{1}$ Department of Mechanical Engineering, Mahanakorn University of Technology, Bangkok, Thailand \\ ${ }^{2}$ Department of Mechanical Engineering, Chulalongkorn University, Bangkok, Thailand \\ Email: *nuttawit_mut@hotmail.com, v_ungbhakorn@yahoo.com
}

Received August 24, 2012; revised September 25, 2012; accepted October 10, 2012

\begin{abstract}
The differential transformation method (DTM) is applied to investigate free vibration of functionally graded beams supported by arbitrary boundary conditions, including various types of elastically end constraints. The material properties of functionally graded beams are assumed to obey the power law distribution. The main advantages of this method are known for its excellence in high accuracy with small computational expensiveness. The DTM also provides all natural frequencies and mode shapes without any frequency missing. Fundamental frequencies as well as their higher frequencies and mode shapes are presented. The significant aspects such as boundary conditions, values of translational and rotational spring constants and the material volume fraction index on the natural frequencies and mode shapes are discussed. For elastically end constraints, some available results of special cases for isotropic beams are used to validate the present results. The new frequency results and mode shapes of functionally graded beams resting on elastically end constraints are presented.
\end{abstract}

Keywords: Functionally Graded Beams; Free Vibration; Natural Frequency; Mode Shape; Differential Transformation Method

\section{Introduction}

A new class of composite materials, called functionally graded materials (FGMs), is considered in this paper. The potential uses of FGMs in engineering applications include aerospace structures, engine combustion chambers, fusion energy devices, engine parts and other engineering structures. In recent years, the static and dynamic analyses of functionally graded (FG) beams have increasingly attracted many researchers.

Sankar [1] provided an elasticity solution based on Euler-Bernoulli beam theory for bending analysis of FG beams. An analytical solution of cantilever FG beams subjected to various types of loadings was presented by Zhong and $\mathrm{Yu}$ [2] using the Airy stress function. Deflection and natural frequency results of the layered FG beams were obtained from the zigzag theoretical modeling and experiment by Kapuria et al. [3]. Sina et al. [4] employed the first order shear deformation (FSDT) to solve the free vibration problem of FG beams. The Lagrange multiplier method was used to solve the fundamental frequency of FG beams based on different higherorder beam theories in the study of Simsek [5]. Free vibration analysis of simply supported FG beams was done

${ }^{*}$ Corresponding author. by Aydogdu and Taskin [6]. Wattanasakulpong et al. [7] used an improved third order shear deformation theory to analyze free vibration of FG beams using the Ritz method. The finite element method was used by Alshorbagy et al. [8] to investigate free vibration characteristics of FG beams. Yang and Chen [9] provided analytical solution based on classical beam theory (CBT) for investigating natural frequencies and critical buckling load of FG beams with edge cracks. The edge cracked FG beams were also studied by Kitipornchai et al. [10] using Timoshenko beam theory. The problems of FG beams resting on elastic foundations were solved by Sahraee et al. [11] and Pradhan and Murmu [12] using differential quadrature method (DQM). Isotropic beams, supported by elastically end constraints, were the subject of many researchers [13-16], but none is dealing with FG beams.

Differential transformation method (DTM) based on Taylor series expansion was initially introduced by Zhou [17] in his study of electrical circuits. It was first implemented to solve vibration analysis of beams by Malik and Dang [18]. Kaya and Ozgumus [19-21] successfully used the DTM to solve many cases of vibration problems. Pradhan et al. [22] also used the DTM to solve the buckling problem of a single walled carbon nanotube.

According to the authors' knowledge, there is no pre- 
vious study on free vibration of FG beams supported by elastically end constraints in the open literature. In this study, the effective tool, DTM, is implemented to analyze free vibration of FG beams with arbitrary boundary conditions, including various types of elastically end constraints. Fundamental frequencies as well as their higher frequencies and mode shapes are presented. The effects of spring constants and the material volume fraction index on the natural frequencies and mode shapes are discussed. Some available special cases in the open literature are used to validate the present results derived from DTM.

\section{Functionally Graded Materials}

A functionally graded beam made of ceramic-metal is considered in this study. The beam geometry and the variation of material volume fraction across the beam thickness associated with the power law distribution are shown in Figure 1.

Based on the rule of mixture, the effective material properties, $P$, can be written as

$$
P=P_{m} V_{m}+P_{c} V_{c}
$$

where $P_{m}, P_{c}, V_{m}$ and $V_{c}$ are material properties and the volume fraction of the metal and ceramic respectively with the relation

$$
V_{m}+V_{c}=1 .
$$

According to the power law distribution, the volume fraction of ceramic can be written as

$$
V_{c}=\left(\frac{z}{h}+\frac{1}{2}\right)^{n}
$$

where the positive number, $0 \leq n \leq \infty$, is the power law or volume fraction index. The FG beam becomes a fully ceramic beam when $n$ is set to zero. From the above relationship, the material properties, in terms of Young's modulus and mass density are expressed as

$$
\begin{aligned}
& E(z)=\left(E_{c}-E_{m}\right)\left(\frac{z}{h}+\frac{1}{2}\right)^{n}+E_{m}, \\
& \rho(z)=\left(\rho_{c}-\rho_{m}\right)\left(\frac{z}{h}+\frac{1}{2}\right)^{n}+\rho_{m} .
\end{aligned}
$$

Delale and Erdogan [23] indicated that the effect of Poisson's ratio on the behavior of the FG plate is much less than that of the Young's modulus, thus the Poisson's ratio will assume to be constant in our study.

\section{The FGM Beam Vibration Analysis}

Consider a classical beam theory (CBT) based on the Kirchhoff-Love hypothesis, the displacements of an arbitrary point along $x$ and $z$ axes can be expressed as follows:

$$
u(x, z, t)=u_{0}-z \frac{\partial w}{\partial x}, w(x, z, t)=w_{0}(x, t) .
$$

where $u_{0}$ and $w_{0}$ are the displacements at a point in the mid-plane. From the displacements in Equation (5), one can obtain the non-zero strains of the beam as

$$
\varepsilon_{x x}=\frac{\partial u_{0}}{\partial x}-z \frac{\partial^{2} w}{\partial x^{2}}=\varepsilon_{0}-z \xi_{x} ; \varepsilon_{0}=\frac{\partial u_{0}}{\partial x}, \xi_{x}=\frac{\partial^{2} w}{\partial x^{2}} .
$$

The normal force resultant, $N_{x}$, moment resultant, $M_{x}$, and transverse shear force, $Q_{x}$, take the form:

$$
\begin{gathered}
N_{x}=A_{11} \varepsilon_{0}-B_{11} \xi_{x}, \\
M_{x}=B_{11} \varepsilon_{0}-D_{11} \xi_{x}, \\
Q_{x}=\frac{\partial M_{x}}{\partial x}=B_{11} \frac{\partial \varepsilon_{0}}{\partial x}-D_{11} \frac{\partial \xi_{x}}{\partial x},
\end{gathered}
$$

where

$$
\left(A_{11}, B_{11}, D_{11}\right)=\int_{-h / 2}^{h / 2} \frac{E(z)}{\left[1-v^{2}\right]}\left(1, z, z^{2}\right) \mathrm{d} z .
$$

The extensional stiffness $\left(A_{11}\right)$, extensional-bending

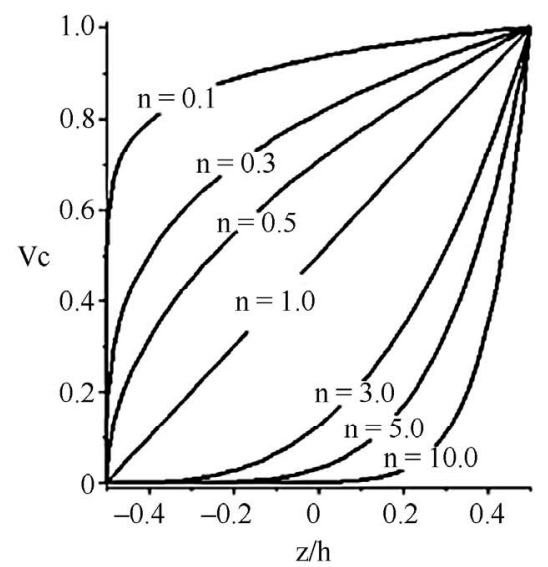

Figure 1. Geometry of a functionally graded beam and volume fraction profile. 
coupling stiffness $\left(B_{11}\right)$ and bending stiffness $\left(D_{11}\right)$ can be written in the function of the volume faction index (n) as:

$$
\begin{gathered}
A_{11}=\frac{h}{1-v^{2}}\left[\frac{\left(E_{c}-E_{m}\right)}{(n+1)}+E_{m}\right] \\
B_{11}=\frac{\left(E_{c}-E_{m}\right) h^{2}}{1-v^{2}}\left[\frac{n}{2(n+1)(n+2)}\right] \\
D_{11}=\frac{h^{3}}{1-v^{2}}\left[\frac{\left(E_{c}-E_{m}\right)\left(n^{2}+n+2\right)}{4(n+1)(n+2)(n+3)}+\frac{E_{m}}{12}\right]
\end{gathered}
$$

The axial inertia term is neglected, therefore, the governing equations of the FG beams derived from Hamilton's principle can be expressed as follows:

$$
\begin{gathered}
\delta u_{0}: \frac{\partial N_{x}}{\partial x}=0 \rightarrow A_{11} \frac{\partial^{2} u_{0}}{\partial x^{2}}-B_{11} \frac{\partial^{3} w}{\partial x^{3}}=0 \\
\delta w: \frac{\partial^{2} M_{x}}{\partial x}+I_{0} \frac{\partial^{2} w}{\partial t^{2}}=0 \rightarrow\left(D_{11}-\frac{B_{11}^{2}}{A_{11}}\right) \frac{\partial^{4} w}{\partial x^{4}}+I_{0} \frac{\partial^{2} w}{\partial t^{2}}=0 .
\end{gathered}
$$

Substituting harmonic vibration mode, $w=W \mathrm{e}^{\mathrm{i} \omega t}$, into Equation (11) leads to a time independent governing equation as follows:

$$
\left(D_{11}-\frac{B_{11}^{2}}{A_{11}}\right) \frac{\partial^{4} W}{\partial x^{4}}-I_{0} \omega^{2} W=0 .
$$

where $\omega$ is a natural frequency and $I_{0}=\int_{-h / 2}^{h / 2} \rho(z) \mathrm{d} z$ is the moment of inertia which can be expressed in term of the volume faction index as:

$$
I_{0}=h\left[\frac{\left(\rho_{c}-\rho_{m}\right)}{(n+1)}+\rho_{m}\right] .
$$

\section{Application of DTM to FG Beam Vibration Analysis}

The principle of the DTM is to transform the governing differential and boundary condition equations into a set of algebraic equations using transformation rules. The basic operations required in differential transformation for the governing differential and boundary condition equations are shown in Tables $\mathbf{1}$ and $\mathbf{2}$ respectively.

The general function, $f(x)$ in Tables $\mathbf{1}$ and $\mathbf{2}$ is considered as the transverse displacement $W(x)$. Apply the basic operations of DTM in Table 1 with the fundamentals of the DTM presented in [18] to the governing differential equation, Equation (12), one can obtain the recurrence equation as:

$$
W[r+4]=\frac{I_{0} \omega^{2}}{\lambda(r+1)(r+2)(r+3)(r+4)} W[r]
$$

where $\lambda=\left(D_{11}-\frac{B_{11}^{2}}{A_{11}}\right)$.

It is seen that Equation (14) is independent from boundary conditions. Therefore to obtain frequency results, the displacement function of Equation (14) must be used to satisfy the corresponding boundary equations.

\subsection{FG beams without Elastically End Constraints}

Three types of general edge conditions, without any springs, at $x=0$ and $L$ are: Simply supported (S),

Table 1. Basic operations of DTM for the governing equations.

\begin{tabular}{cc}
\hline Original functions & Transformed functions \\
\hline$f(x)=g(x) \pm h(x)$ & $F[r]=G[r] \pm H[r]$ \\
$f(x)=\lambda g(x)$ & $F[r]=\lambda G[r]$ \\
$f(x)=g(x) h(x)$ & $F[r]=\sum_{l=0}^{r} G[r-l] H[l]$ \\
$f(x)=\frac{\mathrm{d}^{p} g(x)}{\mathrm{d} x^{p}}$ & $F[r]=\frac{(r+p) !}{r !} G[r+p]$ \\
$f(x)=x^{p}$ & $F[r]=\delta(r-p)= \begin{cases}0 & r \neq p \\
1 & r=p\end{cases}$ \\
\hline
\end{tabular}

Table 2. Basic operations of DTM for the boundary conditions.

\begin{tabular}{cccc}
\hline & $x=0$ & & $x=L$ \\
\hline Original B.C. & Transformed B.C. & Original B.C. & Transformed B.C. \\
\hline$f(0)=0$ & $F(0)=0$ & $f(L)=0$ & $\sum_{r=0}^{\infty} L^{(r)} F(r)=0$ \\
$\frac{\mathrm{d} f(0)}{\mathrm{d} x}=0$ & $F(1)=0$ & $\frac{\mathrm{d} f(L)}{\mathrm{d} x}=0$ & $\sum_{r=0}^{\infty} r L^{(r-1)} F(r)=0$ \\
$\frac{\mathrm{d}^{2} f(0)}{\mathrm{d} x^{2}}=0$ & $F(2)=0$ & $\frac{\mathrm{d}^{2} f(L)}{\mathrm{d} x^{2}}=0$ & $\sum_{r=0}^{\infty} r(r-1) L^{(r-2)} F(r)=0$ \\
$\frac{\mathrm{d}^{3} f(0)}{\mathrm{d} x^{3}}=0$ & $F(3)=0$ & $\frac{\mathrm{d}^{3} f(L)}{\mathrm{d} x^{3}}=0$ & $\sum_{r=0}^{\infty} r(r-1)(r-2) L^{(r-3)} F(r)=0$ \\
\hline
\end{tabular}


$W=0, \frac{\mathrm{d}^{2} W}{\mathrm{~d} x^{2}}=0 ;$ Clamped (C), $W=0, \frac{\mathrm{d} W}{\mathrm{~d} x}=0$; and

Free $(\mathrm{F}), \frac{\mathrm{d}^{2} W}{\mathrm{~d} x^{2}}=0, \frac{\mathrm{d}^{3} W}{\mathrm{~d} x^{3}}=0$. Now consider a beam with free-free (F-F) boundary conditions. The bending moment and shear force at $x=0$ and $L$ are zero. Let the non-zero values of deflection and slope at $x=0$ indicate by $C_{0}$ and $C_{1}$ respectively. Applying the basic operations of DTM for the boundary condition at $x=0$, using Table 2, one obtains

$$
W[0]=C_{0}, W[1]=C_{1}, W[2]=0, W[3]=0 .
$$

Substituting Equation (15) into the recurrence equation Equation (14) leads to $W[r]$ for all values of $r$ as follows:

$$
\begin{gathered}
W[4 r]=\frac{\omega^{2 r} I_{0}^{r}}{\lambda^{r}(4 r) !} C_{0} \quad r=0,1,2,3, \cdots \\
W[4 r+1]=\frac{\omega^{2 r} I_{0}^{r}}{\lambda^{r}(4 r+1) !} C_{1} \quad r=0,1,2,3, \cdots \\
W[4 r+2]=0 \quad r=0,1,2,3, \cdots \\
W[4 r+3]=0 \quad r=0,1,2,3, \cdots
\end{gathered}
$$

For the boundary condition at $x=L$, applying the basic operations of DTM using Table 2, one obtains

$$
\begin{aligned}
& \sum_{r=0}^{\infty} r(r-1) L^{(r-2)} W[r]=0, \\
& \sum_{r=0}^{\infty} r(r-1)(r-2) L^{(r-3)} W[r]=0
\end{aligned}
$$

Substituting $W[r]$ from Equation (16) into Equation (17) leads to two polynomial equations which can be arranged into the following matrix form.

$$
\left[\begin{array}{ll}
e_{11} & e_{12} \\
e_{21} & e_{22}
\end{array}\right]\left\{\begin{array}{l}
C_{0} \\
C_{1}
\end{array}\right\}=\left\{\begin{array}{l}
0 \\
0
\end{array}\right\}
$$

where

$$
\begin{aligned}
& e_{11}=\sum_{r=1}^{\infty} \frac{\omega^{2 r} I_{0}^{r} L^{(4 r-2)}}{\lambda^{r}(4 r-2) !} ; e_{12}=\sum_{r=1}^{\infty} \frac{\omega^{2 r} I_{0}^{r} L^{(4 r-1)}}{\lambda^{r}(4 r-1) !} ; \\
& e_{21}=\sum_{r=1}^{\infty} \frac{\omega^{2 r} I_{0}^{r} L^{(4 r-3)}}{\lambda^{r}(4 r-3) !} ; e_{22}=\sum_{r=1}^{\infty} \frac{\omega^{2 r} I_{0}^{r} L^{(4 r-2)}}{\lambda^{r}(4 r-2) !} .
\end{aligned}
$$

The frequency results can be determined by setting the determinant of the coefficient matrix of Equation (18) to zero. Hence, the frequency equation can be expressed with the finite number of terms in each component of the matrix from $r$ to $R$ as:

$$
\begin{aligned}
& \sum_{r=1}^{R} \frac{\omega^{2 r} I_{0}^{r} L^{(4 r-2)}}{\lambda^{r}(4 r-2) !} \times \sum_{r=1}^{R} \frac{\omega^{2 r} I_{0}^{r} L^{(4 r-2)}}{\lambda^{r}(4 r-2) !} \\
& -\sum_{r=1}^{R} \frac{\omega^{2 r} I_{0}^{r} L^{(4 r-3)}}{\lambda^{r}(4 r-3) !} \times \sum_{r=1}^{R} \frac{\omega^{2 r} I_{0}^{r} L^{(4 r-1)}}{\lambda^{r}(4 r-1) !}=0
\end{aligned}
$$

Solving the frequency equation in Equation (19), one obtains the frequency results as: $\omega=\omega_{r}^{[R]}$, where $r=1,2$, $3, \cdots, R$. Therefore, $\omega_{r}^{[R]}$ is the $r^{\text {th }}$ estimated frequency corresponding to $R$. Hence, an appropriate value of $R$ is obtained by convergence analysis with the following criterion,

$$
\left|\omega_{r}^{[R]}-\omega_{r}^{[R-1]}\right| \leq \delta
$$

where $\delta$ is a given error tolerance.

The mode shape function can be obtained using $W(x)=\sum_{r=0}^{R} x^{r} W[r]$, that is

$$
\begin{aligned}
W(x)= & \sum_{r=0}^{R} \frac{\omega^{2 r} I_{0}^{r}}{\lambda^{r}(4 r) !} x^{(4 r)} \\
& -\frac{\sum_{r=1}^{R} \frac{\omega^{2 r} I_{0}^{r} L^{(4 r-2)}}{\lambda^{r}(4 r-2) !} \sum_{r=0}^{R} \frac{\omega^{2 r} I_{0}^{r}}{\lambda^{r}(4 r+1) !} x^{(4 r+1)}}{\sum_{r=1}^{R} \frac{\omega^{2 r} I_{0}^{r} L^{(4 r-1}}{\lambda^{r}(4 r-1) !}}
\end{aligned}
$$

Following the same procedure, one can obtain the frequency equation and mode shape function for other kinds of boundary conditions without any spring support as given in Appendix A.

\subsection{FG Beams with Elastically End Constraints}

A FG beam supporting by elastic translational and rotational springs at both ends, called E-E boundary conditions, is shown in Figure 2. For this case, the boundary conditions at the left end can be expressed as,

$$
k_{T L} W+Q_{x}=0, \quad k_{R L} \frac{\mathrm{d} W}{\mathrm{~d} x}-M_{x}=0 .
$$

The boundary conditions in Equation (22) can be take another form as

$$
\frac{\mathrm{d}^{3} W}{\mathrm{~d} x^{3}}+\frac{k_{T L}}{\lambda} W=0, \quad \frac{\mathrm{d}^{2} W}{\mathrm{~d} x^{2}}-\frac{k_{R L}}{\lambda} \frac{\mathrm{d} W}{\mathrm{~d} x}=0 .
$$

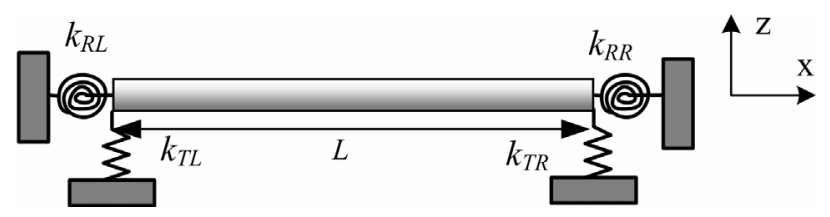

Figure 2. Geometry of FG beam with E-E boundary condition. 
Where $k_{T L}$ and $k_{R L}$ are the translational spring constant $(\mathrm{MN} / \mathrm{m})$ and the rotational spring constant $(\mathrm{MN} \cdot \mathrm{m} / \mathrm{rad})$ at the left end respectively. Let the non-zero values of deflection and slope at $x=0$ be $C_{0}$ and $C_{1}$ respectively. Use Table 2 to apply the basic operations of DTM for these non-zero quantities at $x=0$, one obtains

$$
W[0]=W(x)=C_{0}, \quad W[1]=\frac{\mathrm{d} W(x)}{\mathrm{d} x}=C_{1} .
$$

The expressions for non-zero values of bending moment and shear force at $x=0$ can be written as

$$
W[2]=\frac{k_{R L} C_{1}}{2 \lambda}, W[3]=-\frac{k_{T L} C_{0}}{6 \lambda} .
$$

To find $W[r]$ for all values of $r$, the components in Equations (24) and (25) are substituted into the recurrence Equation (14).

$$
\begin{gathered}
W[4 r]=\frac{\omega^{2 r} I_{0}^{r} C_{0}}{\lambda^{r}(4 r) !}, \quad r=0,1,2,3, \cdots . \\
W[4 r]=\frac{\omega^{2 r} I_{0}^{r} C_{0}}{\lambda^{r}(4 r) !}, \quad r=0,1,2,3, \cdots . \\
W[4 r+2]=\frac{\omega^{2 r} I_{0}^{r} k_{R L} C_{1}}{\lambda^{(r+1)}(4 r+2) !}, \quad r=0,1,2,3, \cdots . \\
W[4 r+3]=-\frac{\omega^{2 r} I_{0}^{r} k_{T L} C_{0}}{\lambda^{(r+1)}(4 r+3) !}, \quad r=0,1,2,3, \cdots .
\end{gathered}
$$

At $x=L$, the boundary conditions are

$$
k_{R R} \frac{\mathrm{d} W}{\mathrm{~d} x}+M_{x}=0, k_{T R} W-Q_{x}=0 .
$$

They can be written as:

$$
\frac{\mathrm{d}^{2} W}{\mathrm{~d} x^{2}}+\frac{k_{R R}}{\lambda} \frac{\mathrm{d} W}{\mathrm{~d} x}=0, \quad \frac{\mathrm{d}^{3} W}{\mathrm{~d} x^{3}}-\frac{k_{T R}}{\lambda} W=0 .
$$

Similarly, applying the DTM to the boundary conditions (28) yields

$$
\begin{gathered}
\sum_{r=0}^{\infty} r(r-1) L^{(r-2)} W[r]+\frac{k_{R R}}{\lambda} \sum_{r=0}^{\infty} r L^{(r-1)} W[r]=0, \\
\sum_{r=0}^{\infty} r(r-1)(r-2) L^{(r-3)} W[r]-\frac{k_{T R}}{\lambda} \sum_{r=0}^{\infty} L^{(r)} W[r]=0
\end{gathered}
$$

Substituting $W[r]$ from Equation (26) into Equation (29) leads to two polynomial equations which can be arranged into the following matrix form:

$$
\left[\begin{array}{ll}
p_{11} & p_{12} \\
p_{21} & p_{22}
\end{array}\right]\left\{\begin{array}{l}
C_{0} \\
C_{1}
\end{array}\right\}=\left\{\begin{array}{l}
0 \\
0
\end{array}\right\}
$$

where:

$$
\begin{aligned}
p_{11}= & \sum_{r=1}^{\infty} \frac{L^{(4 r-2)} \omega^{2 r} I_{0}^{r}}{\lambda^{r}(4 r-2) !}+k_{R R} \sum_{r=1}^{\infty} \frac{L^{(4 r-1)} \omega^{2 r} I_{0}^{r}}{\lambda^{(r+1)}(4 r-1) !} \\
& -k_{T L} \sum_{r=0}^{\infty} \frac{L^{(4 r+1)} \omega^{2 r} I_{0}^{r}}{\lambda^{(r+1)}(4 r+1) !}-k_{R R} k_{T L} \sum_{r=0}^{\infty} \frac{L^{(4 r+2)} \omega^{2 r} I_{0}^{r}}{\lambda^{(r+2)}(4 r+2) !} \\
p_{12}= & \sum_{r=1}^{\infty} \frac{L^{(4 r-1)} \omega^{2 r} I_{0}^{r}}{\lambda^{r}(4 r-1) !}+k_{R R} \sum_{r=0}^{\infty} \frac{L^{(4 r)} \omega^{2 r} I_{0}^{r}}{\lambda^{(r+1)}(4 r) !} \\
& +k_{R L} \sum_{r=0}^{\infty} \frac{L^{(4 r)} \omega^{2 r} I_{0}^{r}}{\lambda^{(r+1)}(4 r) !}+k_{R R} k_{R L} \sum_{r=0}^{\infty} \frac{L^{(4 r+1)} \omega^{2 r} I_{0}^{r}}{\lambda^{(r+2)}(4 r+1) !} \\
p_{21}= & \sum_{r=1}^{\infty} \frac{L^{(4 r-3)} \omega^{2 r} I_{0}^{r}}{\lambda^{r}(4 r-3) !}-k_{T R} \sum_{r=0}^{\infty} \frac{L^{(4 r)} \omega^{2 r} I_{0}^{r}}{\lambda^{(r+1)}(4 r) !} \\
& -k_{T L} \sum_{r=0}^{\infty} \frac{L^{(4 r)} \omega^{2 r} I_{0}^{r}}{\lambda^{(r+1)}(4 r) !}+k_{T R} k_{T L} \sum_{r=0}^{\infty} \frac{L^{(4 r+3)} \omega^{2 r} I_{0}^{r}}{\lambda^{(r+2)}(4 r+3) !} \\
p_{22}= & \sum_{r=1}^{\infty} \frac{L^{(4 r-2)} \omega^{2 r} I_{0}^{r}}{\lambda^{r}(4 r-2) !}-k_{T R} \sum_{r=0}^{\infty} \frac{L^{(4 r+1)} \omega^{2 r} I_{0}^{r}}{\lambda^{(r+1)}(4 r+1) !} \\
+ & k_{R L} \sum_{r=1}^{\infty} \frac{L^{(4 r-1)} \omega^{2 r} I_{0}^{r}}{\lambda^{(r+1)}(4 r-1) !}-k_{T R} k_{R L} \sum_{r=0}^{\infty} \frac{L^{(4 r+2)} \omega^{2 r} I_{0}^{r}}{\lambda^{(r+2)}(4 r+2) !} .
\end{aligned}
$$

Similarly, set the determinant of the coefficient matrix of Equation (30) to zero with finite number of terms, one obtains the following frequency equation.

$$
\begin{aligned}
& {\left[\sum_{r=1}^{R}\left(\frac{L^{(4 r-2)} \omega^{2 r} I_{0}^{r}}{\lambda^{r}(4 r-2) !}+k_{R R} \frac{L^{(4 r-1)} \omega^{2 r} I_{0}^{r}}{\lambda^{(r+1)}(4 r-1) !}\right)-\sum_{r=0}^{R}\left(k_{T L} \frac{L^{(4 r+1)} \omega^{2 r} I_{0}^{r}}{\lambda^{(r+1)}(4 r+1) !}+k_{R R} k_{T L} \frac{L^{(4 r+2)} \omega^{2 r} I_{0}^{r}}{\lambda^{(r+2)}(4 r+2) !}\right)\right]} \\
& \times\left[\sum_{r=1}^{R}\left(\frac{L^{(4 r-2)} \omega^{2 r} I_{0}^{r}}{\lambda^{r}(4 r-2) !}+k_{R L} \frac{L^{(4 r-1)} \omega^{2 r} I_{0}^{r}}{\lambda^{(r+1)}(4 r-1) !}\right)-\sum_{r=0}^{R}\left(k_{T R} \frac{L^{(4 r+1)} \omega^{2 r} I_{0}^{r}}{\lambda^{(r+1)}(4 r+1) !}+k_{T R} k_{R L} \frac{L^{(4 r+2)} \omega^{2 r} I_{0}^{r}}{\lambda^{(r+2)}(4 r+2) !}\right)\right] \\
& -\left[\sum_{r=1}^{R} \frac{L^{(4 r-3)} \omega^{2 r} I_{0}^{r}}{\lambda^{r}(4 r-3) !}+\sum_{r=0}^{R}\left(k_{T R} k_{T L} \frac{L^{(4 r+3)} \omega^{2 r} I_{0}^{r}}{\lambda^{(r+2)}(4 r+3) !}-k_{T R} \frac{L^{(4 r)} \omega^{2 r} I_{0}^{r}}{\lambda^{(r+1)}(4 r) !}-k_{T L} \frac{L^{(4 r)} \omega^{2 r} I_{0}^{r}}{\lambda^{(r+1)}(4 r) !}\right)\right] \\
& \times\left[\sum_{r=1}^{R} \frac{L^{(4 r-1)} \omega^{2 r} I_{0}^{r}}{\lambda^{r}(4 r-1) !}+\sum_{r=0}^{R}\left(k_{R R} k_{R L} \frac{L^{(4 r+1)} \omega^{2 r} I_{0}^{r}}{\lambda^{(r+2)}(4 r+1) !}+k_{R R} \frac{L^{(4 r)} \omega^{2 r} I_{0}^{r}}{\lambda^{(r+1)}(4 r) !}+k_{R L} \frac{L^{(4 r)} \omega^{2 r} I_{0}^{r}}{\lambda^{(r+1)}(4 r) !}\right)\right]=0
\end{aligned}
$$


The mode shape function corresponding to the frequency in Equation (31) can be derived as:

$$
\begin{aligned}
& W(x) \\
= & {\left[\sum_{r=0}^{R} \frac{\omega^{2 r} I_{0}^{r}}{\lambda^{r}(4 r) !} x^{(4 r)}+C_{1} \sum_{r=0}^{R} \frac{\omega^{2 r} I_{0}^{r}}{\lambda^{r}(4 r+1) !} x^{(4 r+1)}\right.} \\
& \left.+C_{1} \sum_{r=0}^{R} \frac{\omega^{2 r} I_{0}^{r} k_{R L}}{\lambda^{(r+1)}(4 r+2) !} x^{(4 r+2)}-\sum_{r=0}^{R} \frac{\omega^{2 r} I_{0}^{r} k_{T L}}{\lambda^{(r+1)}(4 r+3) !} x^{(4 r+3)}\right]
\end{aligned}
$$

where: (please see the Equation (33) below).

Following similar procedure, one can obtain the frequency equation and mode shape function for a clampedelastic supported (C-E) beam in Figure 3 as follows.

The frequency equation:

$$
\begin{aligned}
& {\left[\sum_{r=0}^{R}\left(\frac{L^{(4 r)} \omega^{2 r} I_{0}^{r}}{\lambda^{r}(4 r) !}+k_{R R} \frac{L^{(4 r+1)} \omega^{2 r} I_{0}^{r}}{\lambda^{(r+1)}(4 r+1) !}\right)\right]} \\
& \times\left[\sum_{r=0}^{R}\left(\frac{L^{(4 r)} \omega^{2 r} I_{0}^{r}}{\lambda^{r}(4 r) !}-k_{T R} \frac{L^{(4 r+3)} \omega^{2 r} I_{0}^{r}}{\lambda^{(r+1)}(4 r+3) !}\right)\right] \\
& -\left[\sum_{r=1}^{R} \frac{L^{(4 r-1)} \omega^{2 r} I_{0}^{r}}{\lambda^{r}(4 r-1) !}-k_{T R} \sum_{r=0}^{R} \frac{L^{(4 r+2)} \omega^{2 r} I_{0}^{r}}{\lambda^{(r+1)}(4 r+2) !}\right] \\
& \times\left[\sum_{r=0}^{R}\left(\frac{L^{(4 r+1)} \omega^{2 r} I_{0}^{r}}{\lambda^{r}(4 r+1) !}+k_{R R} \frac{L^{(4 r+2)} \omega^{2 r} I_{0}^{r}}{\lambda^{(r+1)}(4 r+2) !}\right)\right]=0
\end{aligned}
$$

The mode shape function:

$$
\begin{aligned}
W(x)= & \sum_{r=0}^{R} \frac{\omega^{2 r} I_{0}^{r}}{\lambda^{r}(4 r+2) !} x^{(4 r+2)} \\
& +C_{1} \sum_{r=0}^{R} \frac{\omega^{2 r} I_{0}^{r}}{\lambda^{r}(4 r+3) !} x^{(4 r+3)}
\end{aligned}
$$

where:

$$
C_{1}=-\frac{\sum_{r=0}^{R}\left(\frac{L^{(4 r)} \omega^{2 r} I_{0}^{r}}{\lambda^{r}(4 r) !}+k_{R R} \frac{L^{(4 r+1)} \omega^{2 r} I_{0}^{r}}{\lambda^{(r+1)}(4 r+1) !}\right)}{\sum_{r=0}^{R}\left(\frac{L^{(4 r+1)} \omega^{2 r} I_{0}^{r}}{\lambda^{r}(4 r+1) !}+k_{R R} \frac{L^{(4 r+2)} \omega^{2 r} I_{0}^{r}}{\lambda^{(r+1)}(4 r+2) !}\right)} .
$$

For the case of simply supported-elastic (S-E) FG beams as shown in Figure 4, the expressions for the frequency equation and the mode shape function can be written as:

The frequency equation:

$$
\begin{aligned}
& {\left[\sum_{r=1}^{R} \frac{L^{(4 r-1)} \omega^{2 r} I_{0}^{r}}{\lambda^{r}(4 r-1) !}+k_{R R} \sum_{r=0}^{R} \frac{L^{(4 r)} \omega^{2 r} I_{0}^{r}}{\lambda^{(r+1)}(4 r) !}\right]} \\
& \times\left[\sum_{r=0}^{R}\left(\frac{L^{(4 r)} \omega^{2 r} I_{0}^{r}}{\lambda^{r}(4 r) !}-k_{T R} \frac{L^{(4 r+3)} \omega^{2 r} I_{0}^{r}}{\lambda^{(r+1)}(4 r+3) !}\right)\right] \\
& -\left[\sum_{r=1}^{R} \frac{L^{(4 r-2)} \omega^{2 r} I_{0}^{r}}{\lambda^{r}(4 r-2) !}-k_{T R} \sum_{r=0}^{R} \frac{L^{(4 r+1)} \omega^{2 r} I_{0}^{r}}{\lambda^{(r+1)}(4 r+1) !}\right] \\
& \times\left[\sum_{r=0}^{R}\left(\frac{L^{(4 r+1)} \omega^{2 r} I_{0}^{r}}{\lambda^{r}(4 r+1) !}+k_{R R} \frac{L^{(4 r+2)} \omega^{2 r} I_{0}^{r}}{\lambda^{(r+1)}(4 r+2) !}\right)\right]=0
\end{aligned}
$$

The mode shape function:

$$
\begin{aligned}
W(x)= & \sum_{r=0}^{R} \frac{\omega^{2 r} I_{0}^{r}}{\lambda^{r}(4 r+1) !} x^{(4 r+1)} \\
& +C_{1} \sum_{r=0}^{R} \frac{\omega^{2 r} I_{0}^{r}}{\lambda^{r}(4 r+3) !} x^{(4 r+3)}
\end{aligned}
$$

where

$$
C_{1}=-\frac{\sum_{r=1}^{R} \frac{L^{(4 r-1)} \omega^{2 r} I_{0}^{r}}{\lambda^{r}(4 r-1) !}+k_{R R} \sum_{r=0}^{R} \frac{L^{(4 r)} \omega^{2 r} I_{0}^{r}}{\lambda^{(r+1)}(4 r) !}}{\sum_{r=0}^{R}\left(\frac{L^{(4 r+1)} \omega^{2 r} I_{0}^{r}}{\lambda^{r}(4 r+1) !}+k_{R R} \frac{L^{(4 r+2)} \omega^{2 r} I_{0}^{r}}{\lambda^{(r+1)}(4 r+2) !}\right)}
$$

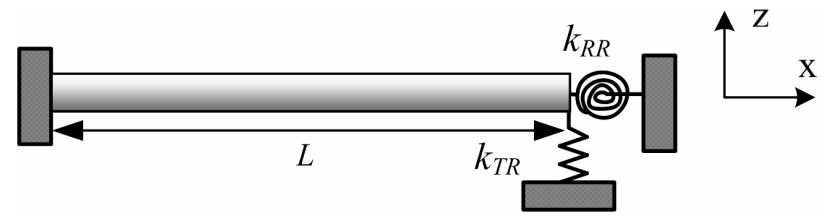

Figure 3. Geometry of FG beam with C-E boundary condition.

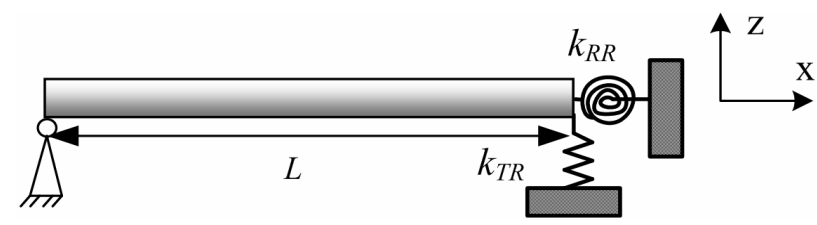

Figure 4. Geometry of FG beam with S-E boundary condition.

$$
C_{1}=-\frac{\sum_{r=1}^{R}\left(\frac{L^{(4 r-2)} \omega^{2 r} I_{0}^{r}}{\lambda^{r}(4 r-2) !}+k_{R R} \frac{L^{(4 r-1)} \omega^{2 r} I_{0}^{r}}{\lambda^{(r+1)}(4 r-1) !}\right)-\sum_{r=0}^{R}\left(k_{T L} \frac{L^{(4 r+1)} \omega^{2 r} I_{0}^{r}}{\lambda^{(r+1)}(4 r+1) !}+k_{R R} k_{T L} \frac{L^{(4 r+2)} \omega^{2 r} I_{0}^{r}}{\lambda^{(r+2)}(4 r+2) !}\right)}{\sum_{r=1}^{R} \frac{L^{(4 r-1)} \omega^{2 r} I_{0}^{r}}{\lambda^{r}(4 r-1) !}+\sum_{r=0}^{R}\left(k_{R R} k_{R L} \frac{L^{(4 r+1)} \omega^{2 r} I_{0}^{r}}{\lambda^{(r+2)}(4 r+1) !}+k_{R R} \frac{L^{(4 r)} \omega^{2 r} I_{0}^{r}}{\lambda^{(r+1)}(4 r) !}+k_{R L} \frac{L^{(4 r)} \omega^{2 r} I_{0}^{r}}{\lambda^{(r+1)}(4 r) !}\right)} .
$$




\section{Numerical Results and Discussions}

\subsection{FG Beams without Elastically End Constraints}

FG beams made of Alumina $\left(\mathrm{Al}_{2} \mathrm{O}_{3}\right)$ and Aluminum (Al); whose material properties are: $E=380 \mathrm{GPa}, \rho=3960$ $\mathrm{kg} / \mathrm{m}^{3}, v=0.3$ for $\mathrm{Al}_{2} \mathrm{O}_{3}$ and $E=70 \mathrm{GPa}, \rho=2702 \mathrm{~kg} / \mathrm{m}^{3}$, $v=0.3$ for $\mathrm{Al}$; are chosen for this study. Six types of boundary conditions are considered as shown in Table 3. The dimensionless frequency is defined as $\Omega=\omega L^{2} / h \sqrt{\left(\rho_{A l} / E_{A l}\right)}$. From convergence study it is found out that $R$ equals to 15 is sufficient for the required accuracy. Using $R$ more than 15 will present the same results for the first to sixth modes. To receive the frequency results that are higher than sixth mode, the value of $R$ more than 15 may be needed. Five modes of vibration with various volume fraction indexes are presented in Table 3.

Only the fundamental frequencies of the work by Simsek [5] for three types of boundary conditions, namely, (S-S), (C-F) and (C-C), are found in the open literature. Very good agreement with the present results for all volume fraction indexes is confirmed as shown in Table 3. It is seen that, for all boundary conditions, all frequencies decrease as volume fraction indexes increase. The $\mathrm{C}-\mathrm{C}$ and F-F frequencies are practically equal and they are the highest of all boundary conditions. Results in the table show that the volume fraction index is one of the most important parameters that have significant impact on the frequency of vibration and therefore, it must be

Table 3. Dimensionless frequencies of $\mathrm{Al}_{2} \mathrm{O}_{3} / \mathrm{Al}$ beams without springs $(L / h=20)$.

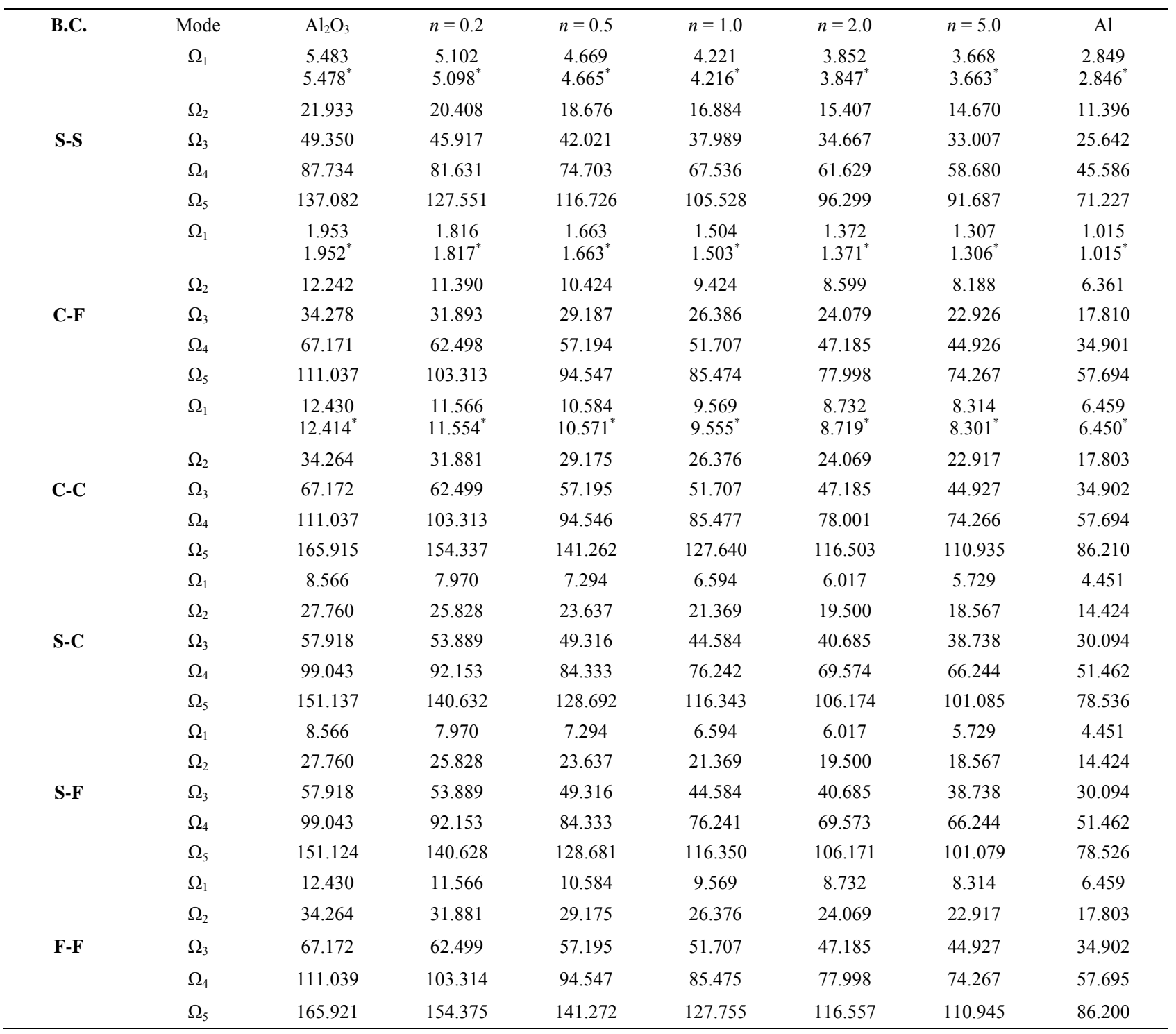

${ }^{*}$ Simsek [5]. 
considered in designing a beam to meet the required frequency. Changing this parameter also means changing flexibility of beams.

\subsection{FG Beams with Elastically End Constraints}

Three types of boundary condition; namely, C-E, S-E and E-E, of FG beams with elastically end constraints will be investigated in the following sections. Due to classical beam theory considered in this study, it is appropriate to choose the thickness ratio $(L / h)$ more than 20 . In the following investigation the ratio $(L / h=30)$ is selected for all of the next calculation. However, the frequency equation and mode shape function presented in this study can be used effectively for other values of the thickness ratio.

\subsubsection{Vibration Analysis of FG Beams with C-E Boundary Condition}

Consider a FG beam completely clamped at the left end and supported by translational and rotational springs at the right end. The beam is defined as the $\mathrm{C}-\mathrm{E}$ beam. The frequency results for the first six modes with various volume fraction indexes are shown in Table 4. To verify the results, only the available work of Lai et al. [14] on the isotropic beam ( $n=0$ for full $\mathrm{Al}_{2} \mathrm{O}_{3}$ ) with the same type of support is shown in the second row. All modes of frequencies agree excellently. Again, all frequencies decrease as volume fraction indexes increase.

Effects of varying the values of spring constants, with $n$ fixed at 0.5 , on the response of FG beams for the first three modes are shown in Table 5. All frequencies increase as spring constants increase as expected. Observe

Table 4. Dimensionless frequencies of $\mathrm{Al}_{2} \mathrm{O}_{3} / \mathrm{Al}$ beams $\left(L / h=30, k_{T R}=1.173 \mathrm{MN} / \mathrm{m}\right.$ and $\left.k_{R R}=1.056 \times 10^{3} \mathrm{MN} \cdot \mathrm{m} / \mathrm{rad}\right)$.

\begin{tabular}{|c|c|c|c|c|c|c|}
\hline & $\Omega_{1}$ & $\Omega_{2}$ & $\Omega_{3}$ & $\Omega_{4}$ & $\Omega_{5}$ & $\Omega_{6}$ \\
\hline$n=0$ & 2.566 & 13.213 & 35.270 & 68.193 & 112.077 & 166.941 \\
\hline Lai et al. [14] & 2.566 & 13.213 & 35.270 & 68.193 & 112.078 & 166.923 \\
\hline$n=0.2$ & 2.547 & 12.970 & 34.493 & 66.611 & 109.420 & 162.902 \\
\hline$n=0.5$ & 2.429 & 12.056 & 31.780 & 61.192 & 100.378 & 149.361 \\
\hline$n=1.0$ & 2.305 & 11.112 & 28.987 & 55.605 & 91.051 & 135.293 \\
\hline$n=2.0$ & 2.206 & 10.343 & 26.707 & 51.033 & 83.400 & 123.834 \\
\hline$n=5.0$ & 2.171 & 9.987 & 25.611 & 48.803 & 79.643 & 118.170 \\
\hline$n=10.0$ & 2.146 & 9.768 & 24.957 & 47.482 & 77.423 & 114.786 \\
\hline
\end{tabular}

Table 5. Dimensionless frequencies of $\mathrm{Al}_{2} \mathrm{O}_{3} / \mathrm{Al}$ beams $(n=0.5 ; L / h=30)$.

\begin{tabular}{|c|c|c|c|c|c|c|c|c|}
\hline \multirow{2}{*}{$(\mathrm{MN} / \mathrm{m})$} & & \multicolumn{7}{|c|}{$k_{R R}(\mathrm{MN} \cdot \mathrm{m} / \mathrm{rad})$} \\
\hline & & 1 & 10 & $10^{2}$ & $10^{3}$ & $10^{4}$ & $10^{5}$ & $10^{6}$ \\
\hline \multirow{3}{*}{$k_{T R}=1$} & $\Omega_{1}$ & 2.051 & 2.056 & 2.102 & 2.383 & 2.788 & 2.891 & 2.903 \\
\hline & $\Omega_{2}$ & 10.983 & 10.995 & 11.113 & 12.006 & 14.116 & 14.898 & 14.997 \\
\hline & $\Omega_{3}$ & 30.617 & 30.628 & 30.745 & 31.726 & 35.036 & 36.755 & 36.994 \\
\hline \multirow{3}{*}{$k_{T R}=10$} & $\Omega_{1}$ & 3.669 & 3.670 & 3.678 & 3.733 & 3.826 & 3.853 & 3.856 \\
\hline & $\Omega_{2}$ & 11.493 & 11.505 & 11.613 & 12.430 & 14.362 & 15.085 & 15.177 \\
\hline & $\Omega_{3}$ & 30.793 & 30.805 & 30.920 & 31.890 & 35.144 & 36.831 & 37.067 \\
\hline \multirow{3}{*}{$k_{T R}=10^{2}$} & $\Omega_{1}$ & 6.737 & 6.739 & 6.766 & 6.969 & 7.492 & 7.710 & 7.738 \\
\hline & $\Omega_{2}$ & 16.370 & 16.372 & 16.391 & 16.541 & 16.967 & 17.163 & 17.189 \\
\hline & $\Omega_{3}$ & 32.806 & 32.816 & 32.911 & 33.704 & 36.300 & 37.649 & 37.839 \\
\hline \multirow{3}{*}{$k_{T R}=10^{3}$} & $\Omega_{1}$ & 7.553 & 7.559 & 7.616 & 8.091 & 9.669 & 10.544 & 10.673 \\
\hline & $\Omega_{2}$ & 23.677 & 23.680 & 23.718 & 24.051 & 25.559 & 26.793 & 27.008 \\
\hline & $\Omega_{3}$ & 46.330 & 46.331 & 46.336 & 46.385 & 46.612 & 46.809 & 46.844 \\
\hline \multirow{3}{*}{$k_{T R}=10^{4}$} & $\Omega_{1}$ & 7.637 & 7.644 & 7.705 & 8.213 & 9.932 & 10.897 & 11.038 \\
\hline & $\Omega_{2}$ & 24.674 & 24.680 & 24.736 & 25.248 & 27.718 & 29.845 & 30.217 \\
\hline & $\Omega_{3}$ & 51.235 & 51.240 & 51.293 & 51.786 & 54.615 & 57.896 & 58.574 \\
\hline \multirow{3}{*}{$k_{T R}=10^{5}$} & $\Omega_{1}$ & 7.646 & 7.652 & 7.713 & 8.225 & 9.958 & 10.932 & 11.074 \\
\hline & $\Omega_{2}$ & 24.768 & 24.774 & 24.832 & 25.362 & 27.924 & 30.124 & 30.507 \\
\hline & $\Omega_{3}$ & 51.653 & 51.659 & 51.717 & 52.262 & 55.410 & 59.017 & 59.746 \\
\hline \multirow{3}{*}{$k_{T R}=10^{6}$} & $\Omega_{1}$ & 7.647 & 7.653 & 7.714 & 8.226 & 9.961 & 10.935 & 11.078 \\
\hline & $\Omega_{2}$ & 24.778 & 24.783 & 24.842 & 25.374 & 27.945 & 30.152 & 30.535 \\
\hline & $\Omega_{3}$ & 51.693 & 51.699 & 51.758 & 52.309 & 55.486 & 59.122 & 59.855 \\
\hline
\end{tabular}


that for very large value of $k_{T R}$ and $k_{R R}$, the frequencies, $\Omega_{1}, \Omega_{2}$ and $\Omega_{3}$ approach those of C-C beam in Table 3 .

The first to forth mode shapes of FG beams with $\mathrm{C}-\mathrm{E}$ boundary condition are shown in Figures 5(a)-(d), respectively. It is seen that different values of spring support change the mode shapes of the vibrating beams significantly. Note that for $k_{T R}=k_{R R}=0$, the beam corresponds to a C-F beam and as $k_{T R}=k_{R R} \rightarrow \infty$, a C-C beam is obtained.

\subsubsection{Vibration Analysis of FG Beams with S-E Boundary Condition}

Dimensionless frequency results of FG beams, with S-E boundary conditions as shown in Figure 4, for the first six modes are tabulated in Table 6. To verify the results, again, only the work of Lai et al. [14] on the isotropic beam $(n=0)$ is available as shown in the second row of Table 6. All frequencies, $\Omega_{1}$ to $\Omega_{6}$, agree excellently. It is seen that, all frequencies decrease as volume fraction indexes increase.

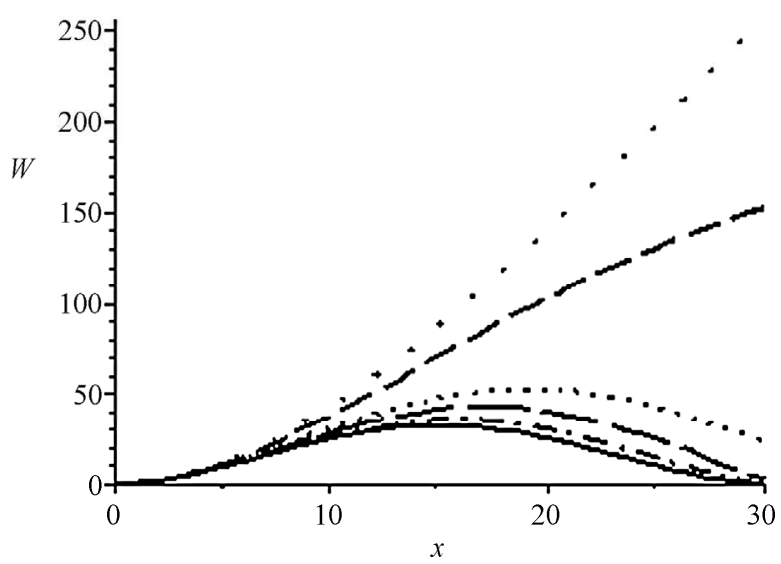

(a)

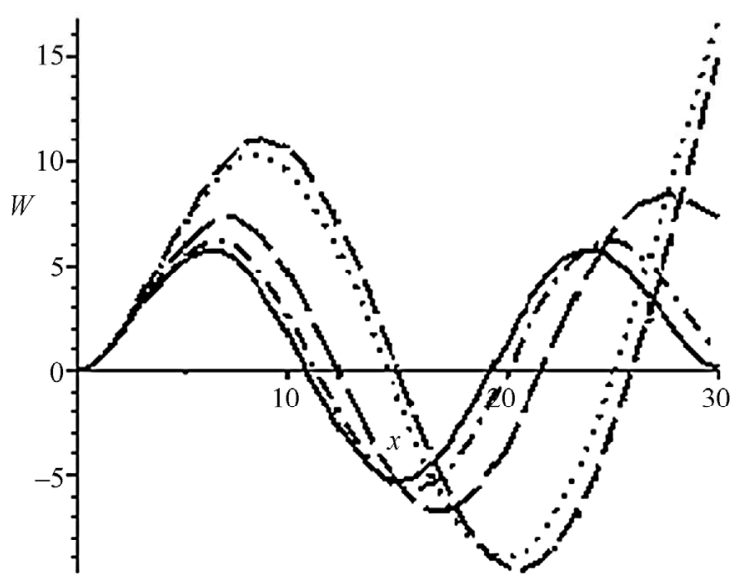

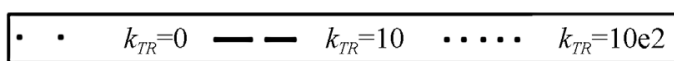

(c)
The first three frequencies for S-E boundary condition with variable spring constants, $k_{T R}$ and $k_{R R}$ are presented in Table 7. To illustrate the effects of spring constants and material volume fraction on the fundamental frequency, 3-D figures for S-E and C-E beams are plotted in Figure 6. And Figure 7 shows the $1^{\text {st }}$ to $4^{\text {th }}$ mode shapes of FG beams with S-E boundary condition.

\subsubsection{Vibration Analysis of FG Beams with E-E Boundary Condition}

FG beams supported by translational and rotational springs at both ends as shown in Figure 2 are considered in this section. Dimensionless frequencies of various modes and volume fraction indexes are presented in Table 8. Again, the accuracy is confirmed by the case of isotropic beams by Lai et al. [14]. It is observed that the first and second frequency results depend mostly on the effects of translational and rotational spring stiffnesses at both ends, Hence, they show different trends of change in comparison with other modes when the value of the

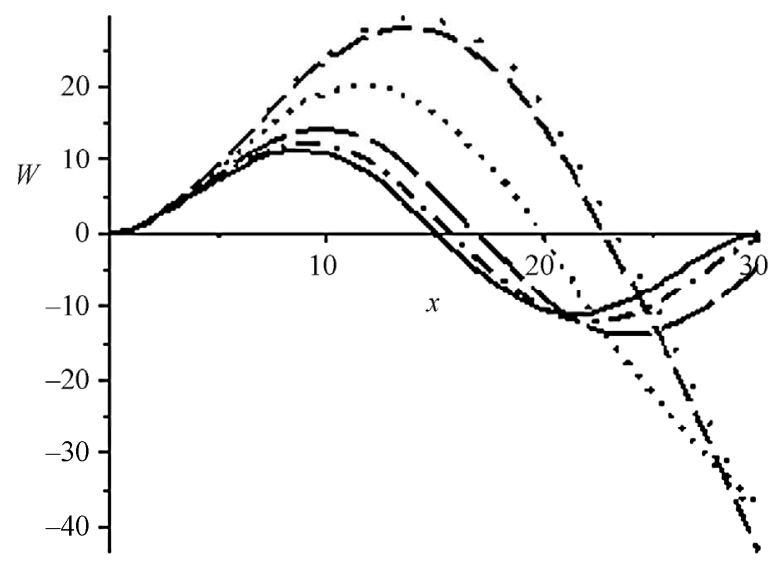

(b)

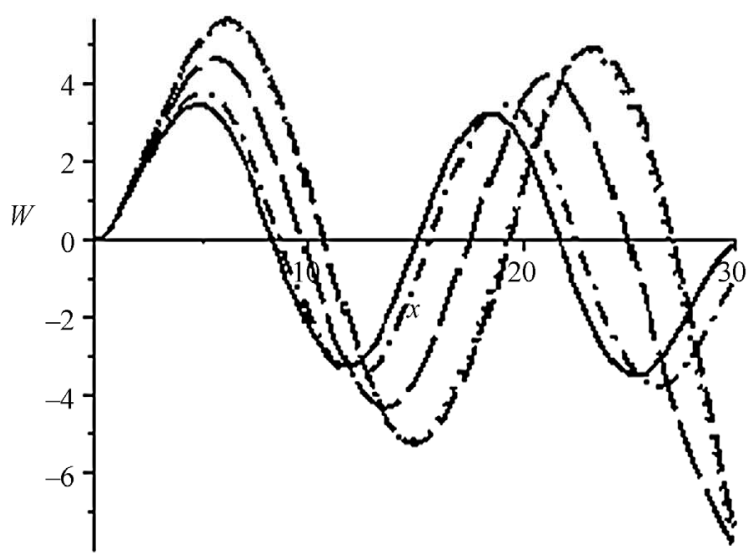

Figure 5. The $1^{\text {st }}$ to $4^{\text {th }}$ mode shapes of $\mathrm{Al}_{2} \mathrm{O}_{3} / \mathrm{Al}$ beams with C-E boundary conditions $\left(n=0.5 ; L / h=30 ; k_{T R}=k_{R R}\right)$. 
Table 6. Dimensionless frequency results of $\mathrm{Al}_{2} \mathrm{O}_{3} / \mathrm{Al}$ beams $\left(L / h=30 ; k_{T R}=29.32 \mathrm{MN} / \mathrm{m} ; k_{R R}=0 \mathrm{MN} \cdot \mathrm{m} / \mathrm{rad}\right)$.

\begin{tabular}{ccccccc}
\hline & $\Omega_{1}$ & $\Omega_{2}$ & $\Omega_{3}$ & $\Omega_{4}$ & $\Omega_{5}$ & $\Omega_{6}$ \\
\hline$n=0$ & 3.826 & 10.471 & 28.340 & 58.189 & 99.200 & 151.238 \\
Lai et al. [14] & 3.826 & 10.471 & 28.340 & 58.189 & 99.200 & 151.238 \\
$n=0.2$ & 3.843 & 10.412 & 27.707 & 56.785 & 96.773 & 147.525 \\
$n=0.5$ & 3.736 & 10.001 & 25.517 & 52.039 & 88.603 & 135.026 \\
$n=1.0$ & 3.576 & 9.616 & 23.284 & 47.142 & 80.156 & 122.113 \\
$n=2.0$ & 3.411 & 9.355 & 21.493 & 43.128 & 73.207 & 111.470 \\
$n=5.0$ & 3.331 & 9.322 & 20.663 & 41.150 & 69.752 & 106.164 \\
$n=10.0$ & 3.273 & 9.282 & 20.176 & 39.986 & 67.718 & 103.042 \\
\hline
\end{tabular}

Table 7. Dimensionless frequencies of $\mathrm{Al}_{2} \mathrm{O}_{3} / \mathrm{Al}$ beams $(n=0.5 ; L / h=30)$.

\begin{tabular}{|c|c|c|c|c|c|c|c|c|}
\hline \multirow{2}{*}{$(\mathrm{MN} / \mathrm{m})$} & & \multicolumn{7}{|c|}{$k_{R R}(\mathrm{MN} \cdot \mathrm{m} / \mathrm{rad})$} \\
\hline & & 1 & 10 & $10^{2}$ & $10^{3}$ & $10^{4}$ & $10^{5}$ & $10^{6}$ \\
\hline \multirow{3}{*}{$k_{T R}=1$} & $\Omega_{1}$ & 0.929 & 0.933 & 0.968 & 1.160 & 1.386 & 1.437 & 1.442 \\
\hline & $\Omega_{2}$ & 7.725 & 7.736 & 7.849 & 8.662 & 10.379 & 10.959 & 11.031 \\
\hline & $\Omega_{3}$ & 24.803 & 24.815 & 24.931 & 25.893 & 28.919 & 30.378 & 30.577 \\
\hline \multirow{3}{*}{$k_{T R}=10$} & $\Omega_{1}$ & 2.652 & 2.652 & 2.652 & 2.653 & 2.654 & 2.655 & 2.655 \\
\hline & $\Omega_{2}$ & 8.455 & 8.464 & 8.558 & 9.239 & 10.707 & 11.216 & 11.279 \\
\hline & $\Omega_{3}$ & 25.022 & 25.034 & 25.148 & 26.093 & 29.048 & 30.471 & 30.665 \\
\hline \multirow{3}{*}{$k_{T R}=10^{2}$} & $\Omega_{1}$ & 4.503 & 4.507 & 4.544 & 4.830 & 5.549 & 5.845 & 5.884 \\
\hline & $\Omega_{2}$ & 13.800 & 13.800 & 13.803 & 13.830 & 13.906 & 13.940 & 13.945 \\
\hline & $\Omega_{3}$ & 27.559 & 27.567 & 27.650 & 28.331 & 30.434 & 31.470 & 31.613 \\
\hline \multirow{3}{*}{$k_{T R}=10^{3}$} & $\Omega_{1}$ & 4.855 & 4.860 & 4.916 & 5.362 & 6.689 & 7.345 & 7.437 \\
\hline & $\Omega_{2}$ & 18.907 & 18.911 & 18.955 & 19.339 & 21.004 & 22.274 & 22.488 \\
\hline & $\Omega_{3}$ & 40.260 & 40.261 & 40.274 & 40.392 & 40.944 & 41.430 & 41.519 \\
\hline \multirow{3}{*}{$k_{T R}=10^{4}$} & $\Omega_{1}$ & 4.891 & 4.897 & 4.954 & 5.418 & 6.818 & 7.517 & 7.615 \\
\hline & $\Omega_{2}$ & 19.513 & 19.519 & 19.576 & 20.088 & 22.404 & 24.226 & 24.532 \\
\hline & $\Omega_{3}$ & 43.716 & 43.722 & 43.776 & 44.278 & 47.059 & 50.075 & 50.673 \\
\hline \multirow{3}{*}{$k_{T R}=10^{5}$} & $\Omega_{1}$ & 4.895 & 4.901 & 4.958 & 5.424 & 6.831 & 7.534 & 7.633 \\
\hline & $\Omega_{2}$ & 19.572 & 19.578 & 19.636 & 20.161 & 22.540 & 24.408 & 24.720 \\
\hline & $\Omega_{3}$ & 44.018 & 44.023 & 44.082 & 44.625 & 47.648 & 50.896 & 51.530 \\
\hline \multirow{3}{*}{$k_{T R}=10^{6}$} & $\Omega_{1}$ & 4.895 & 4.901 & 4.959 & 5.425 & 6.832 & 7.535 & 7.634 \\
\hline & $\Omega_{2}$ & 19.578 & 19.584 & 19.642 & 20.168 & 22.553 & 24.426 & 24.739 \\
\hline & $\Omega_{3}$ & 44.047 & 44.053 & 44.112 & 44.659 & 47.706 & 50.975 & 51.611 \\
\hline
\end{tabular}

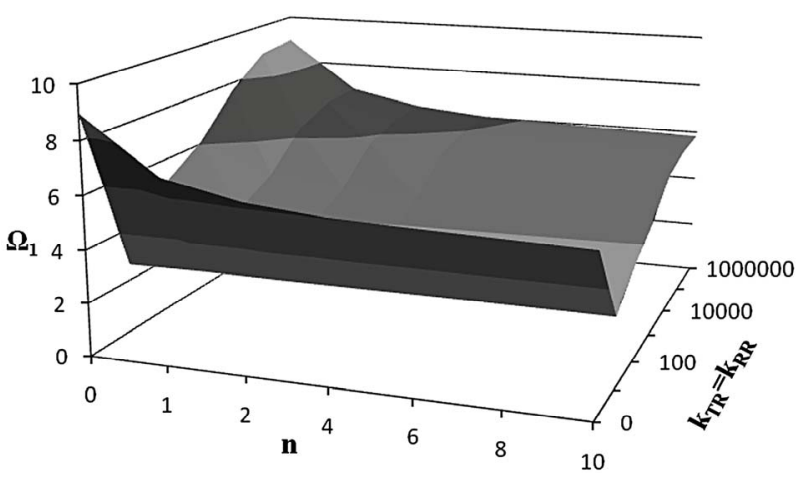

(a) S-E

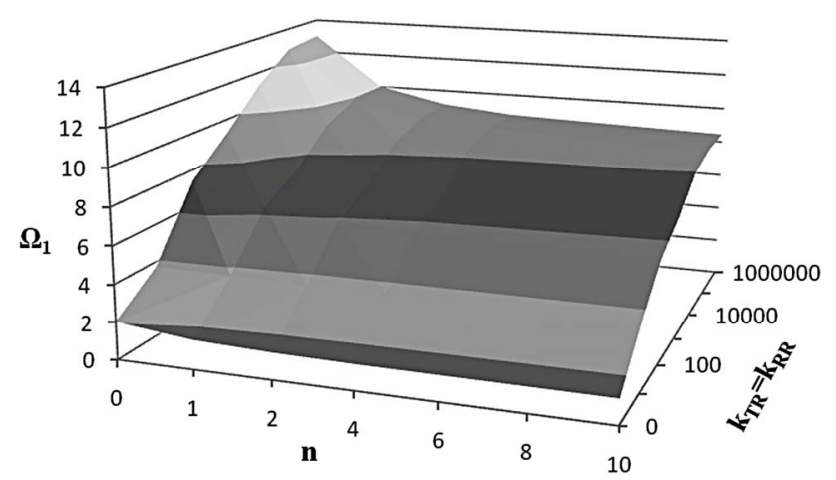

(b) C-E

Figure 6. Fundamental frequency of $\mathrm{Al}_{2} \mathrm{O}_{3} / \mathrm{Al}$ beams with $\mathrm{S}-\mathrm{E}$ and $\mathrm{C}-\mathrm{E}$ boundary conditions $(L / h=30)$. 


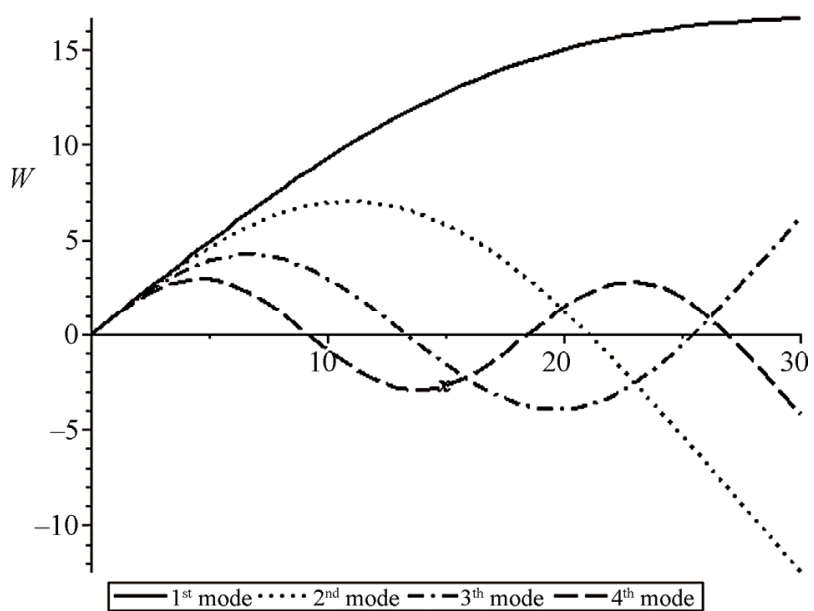

Figure 7. The $1^{\text {st }}$ to $4^{\text {th }}$ mode shapes of $\mathrm{Al}_{2} \mathrm{O}_{3} / \mathrm{Al}$ beams with $\mathrm{S}-\mathrm{E}$ boundary conditions $\left(n=0.5 ; L / h=30 ; k_{T R}=10 \mathrm{MN} / \mathrm{m}\right.$, $\left.k_{R R}=10 \mathrm{MN} \cdot \mathrm{m} / \mathbf{r a d}\right)$. volume fraction index increases. It is clear that as the values of $k_{T L}=k_{T R}=k_{R L}=k_{R R} \rightarrow \infty$, this E-E FG beam behaves like the C-C FG beam (see Table 3).

Table 9 shows the frequency results of the first three modes with variable spring constants. It is similar to the previous cases that frequencies increase as the spring constants increase.

To understand the vibration behavior of FG beams supported by elastically end constraints, the effect of spring constants at both ends on the fundamental frequencies is shown as 3-D plot in Figure 8. Figure 9 shows the $1^{\text {st }}$ to $4^{\text {th }}$ mode shapes of E-E beams. It is observed that the first mode shape depends mostly on the translational springs which move up and down, including a small bending along the length of the beam. But for the second mode shape, it seems to be dependent on the rotational springs in which the movement is clockwise and

Table 8. Dimensionless frequencies of $\mathrm{Al}_{2} \mathrm{O}_{3} / \mathrm{Al}$ beams $\left(L / h=30 ; k_{T L}=k_{T R}=1.173 \mathrm{MN} / \mathrm{m} ; k_{R L}=k_{R R}=1.056 \times 10^{3} \mathrm{MN} \cdot \mathrm{m} / \mathrm{rad}\right)$.

\begin{tabular}{|c|c|c|c|c|c|c|}
\hline & $\Omega_{1}$ & $\Omega_{2}$ & $\Omega_{3}$ & $\Omega_{4}$ & $\Omega_{5}$ & $\Omega_{6}$ \\
\hline$n=0$ & 0.781 & 2.771 & 14.243 & 36.242 & 69.209 & 113.112 \\
\hline Lai et al. [14] & 0.781 & 2.771 & 14.242 & - & - & - \\
\hline$n=0.2$ & 0.802 & 2.822 & 14.046 & 35.532 & 67.700 & 110.531 \\
\hline$n=0.5$ & 0.825 & 2.838 & 13.206 & 32.943 & 62.419 & 101.637 \\
\hline$n=1.0$ & 0.849 & 2.838 & 12.343 & 30.290 & 56.994 & 92.486 \\
\hline$n=2.0$ & 0.876 & 2.833 & 11.653 & 28.148 & 52.588 & 85.025 \\
\hline$n=5.0$ & 0.906 & 2.858 & 11.364 & 27.163 & 50.494 & 81.420 \\
\hline$n=10.0$ & 0.920 & 2.861 & 11.178 & 26.566 & 49.245 & 79.284 \\
\hline
\end{tabular}

Table 9. Dimensionless frequencies of $\mathrm{Al}_{2} \mathrm{O}_{3} / \mathrm{Al}$ beams $(n=0.5 ; L / h=30)$.

\begin{tabular}{|c|c|c|c|c|c|c|c|c|}
\hline \multirow{2}{*}{\multicolumn{2}{|c|}{$(\mathrm{MN} / \mathrm{m}) k_{T L}=k_{T R}$}} & \multicolumn{7}{|c|}{$k_{R L}=k_{R R}(\mathrm{MN} \cdot \mathrm{m} / \mathrm{rad})$} \\
\hline & & 1 & 10 & $10^{2}$ & $10^{3}$ & $10^{4}$ & $10^{5}$ & $10^{6}$ \\
\hline \multirow{3}{*}{1} & $\Omega_{1}$ & 0.760 & 0.760 & 0.760 & 0.762 & 0.765 & 0.766 & 0.766 \\
\hline & $\Omega_{2}$ & 1.330 & 1.355 & 1.582 & 2.746 & 4.439 & 4.945 & 5.006 \\
\hline & $\Omega_{3}$ & 11.204 & 11.226 & 11.444 & 13.112 & 17.442 & 19.325 & 19.579 \\
\hline \multirow{3}{*}{10} & $\Omega_{1}$ & 2.208 & 2.209 & 2.218 & 2.275 & 2.361 & 2.384 & 2.386 \\
\hline & $\Omega_{2}$ & 4.144 & 4.150 & 4.212 & 4.658 & 5.596 & 5.924 & 5.966 \\
\hline & $\Omega_{3}$ & 12.144 & 12.163 & 12.355 & 13.842 & 17.836 & 19.611 & 19.852 \\
\hline \multirow{3}{*}{$10^{2}$} & $\Omega_{1}$ & 4.213 & 4.221 & 4.293 & 4.823 & 6.031 & 6.487 & 6.545 \\
\hline & $\Omega_{2}$ & 11.536 & 11.536 & 11.536 & 11.538 & 11.543 & 11.546 & 11.546 \\
\hline & $\Omega_{3}$ & 19.378 & 19.384 & 19.445 & 19.952 & 21.611 & 22.494 & 22.621 \\
\hline \multirow{3}{*}{$10^{3}$} & $\Omega_{1}$ & 4.816 & 4.828 & 4.937 & 5.811 & 8.529 & 10.070 & 10.305 \\
\hline & $\Omega_{2}$ & 18.314 & 18.323 & 18.406 & 19.133 & 22.115 & 24.313 & 24.683 \\
\hline & $\Omega_{3}$ & 37.532 & 37.535 & 37.565 & 37.832 & 39.012 & 39.962 & 40.127 \\
\hline \multirow{3}{*}{$10^{4}$} & $\Omega_{1}$ & 4.888 & 4.899 & 5.014 & 5.936 & 8.922 & 10.705 & 10.983 \\
\hline & $\Omega_{2}$ & 19.450 & 19.461 & 19.575 & 20.585 & 25.188 & 29.146 & 29.864 \\
\hline & $\Omega_{3}$ & 43.392 & 43.403 & 43.509 & 44.497 & 49.920 & 56.023 & 57.300 \\
\hline \multirow{3}{*}{$10^{5}$} & $\Omega_{1}$ & 4.895 & 4.907 & 5.022 & 5.949 & 8.963 & 10.772 & 11.054 \\
\hline & $\Omega_{2}$ & 19.566 & 19.578 & 19.695 & 20.736 & 25.521 & 29.675 & 30.430 \\
\hline & $\Omega_{3}$ & 43.986 & 43.997 & 44.114 & 45.196 & 51.223 & 58.101 & 59.537 \\
\hline \multirow{3}{*}{$10^{6}$} & $\Omega_{1}$ & 4.896 & 4.907 & 5.023 & 5.951 & 8.967 & 10.779 & 11.061 \\
\hline & $\Omega_{2}$ & 19.578 & 19.589 & 19.707 & 20.751 & 25.555 & 29.728 & 30.486 \\
\hline & $\Omega_{3}$ & 44.044 & 44.056 & 44.174 & 45.265 & 51.352 & 58.304 & 59.754 \\
\hline
\end{tabular}




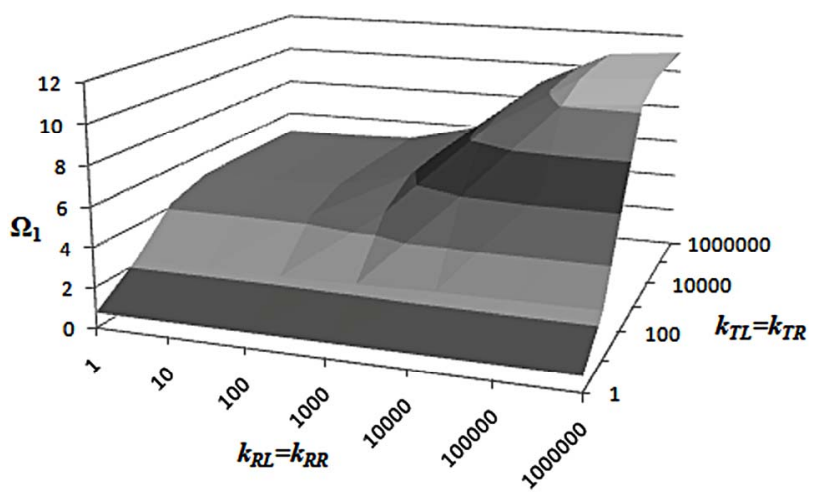

Figure 8. The fundamental frequency of $\mathrm{Al}_{2} \mathrm{O}_{3} / \mathrm{Al}$ beams with $\mathrm{E}-\mathrm{E}$ boundary condition $(n=0.5 ; L / h=30)$.

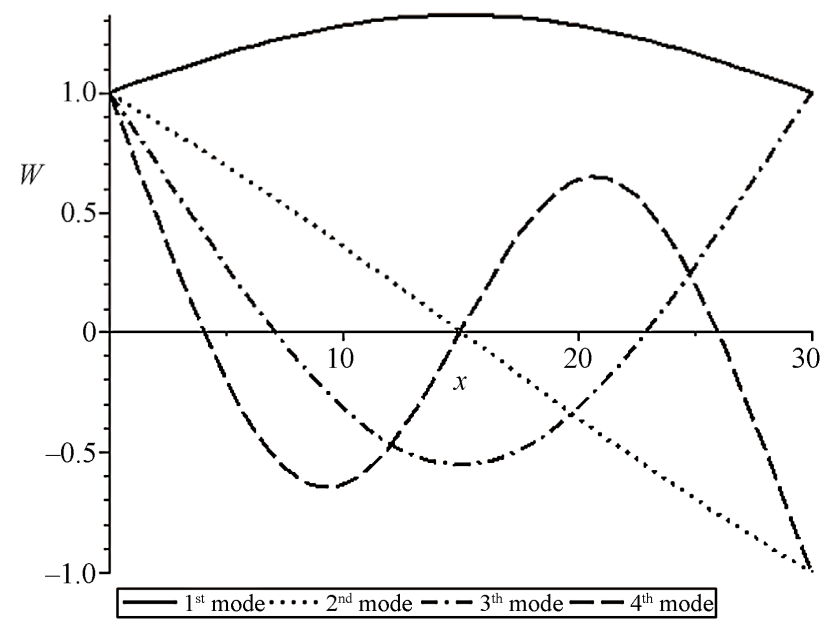

Figure 9. The $1^{\text {st }}$ to $4^{\text {th }}$ mode shape of $\mathrm{Al}_{2} \mathrm{O}_{3} / \mathrm{Al}$ beams with E-E boundary conditions $\left(n=0.5 ; L / h=30 ; k_{T R}=10 \mathrm{MN} / \mathrm{m}\right.$; $\left.k_{R R}=10 \mathrm{MN} \cdot \mathrm{m} / \mathrm{rad}\right)$.

anti-clockwise. If the spring constant becomes large, the mode shapes of E-E beams behave like $\mathrm{C}-\mathrm{C}$ beams.

\section{Concluding Remarks}

This research applies the differential transformation method to solve the governing differential equation of free vibration of functionally graded beams supported by various types of general boundary conditions, including elasticcally end constraints. $\mathrm{FG}$ beams made of $\mathrm{Al}_{2} \mathrm{O}_{3} / \mathrm{Al}$ are chosen to study the free vibration behavior. In general, the results revealed that trend of frequency results for various modes of vibration decreases as the volume fraction indexes increase, except for the case of the E-E boundary conditions in which the trend of the first two modes is reversed owning to the effects of translational and rotational springs at both ends. It is also seen that there are considerable changes of frequencies as well as mode shapes when the stiffness of spring becomes larger. The frequency equation and mode function presented in this study can be specialized to approximate any other boundary conditions, with or without springs, by setting the values of spring constants as appropriate.

\section{REFERENCES}

[1] B. V. Sankar, "An Elasticity Solution for Functionally Graded Beams," Composites Science and Technology, Vol. 61, No. 5, 2001, pp. 689-696. doi:10.1016/S0266-3538(01)00007-0

[2] Z. Zhong and T. Yu, "Analytical Solution of a Cantilever Functionally Graded Beam," Composites Science and Technology, Vol. 67, No. 3-4, 2007, pp. 481-488. doi:10.1016/j.compscitech.2006.08.023

[3] S. Kapuria, M. Bhattacharyya and A. N. Kumar, "Bending and Free Vibration Response of Layered Functionally Graded Beams: A Theoretical Model and Its Experimental Validation," Composite Structures, Vol. 82, No. 3, 2008, pp. 390-402. doi:10.1016/j.compstruct.2007.01.019

[4] S. A. Sina, H. M. Navazi and H. Haddadpour, "An Analytical Method for Free Vibration Analysis of Functionally Graded Beams," Materials \& Design, Vol. 30, No. 3, 2009, pp. 741-747. doi:10.1016/j.matdes.2008.05.015

[5] M. Simsek, "Fundamental Frequency Analysis of Functionally Graded Beams by Using Different Higher-Order Beam Theories," Nuclear Engineering and Design, Vol. 240, No. 4, 2010, pp. 697-705.

doi:10.1016/j.nucengdes.2009.12.013

[6] M. Aydogdu and V. Taskin, "Free Vibration Analysis of Functionally Graded Beams with Simply Supported Edges," Materials \& Design, Vol. 28, No. 5, 2007, pp. 16511656. doi:10.1016/j.matdes.2006.02.007

[7] N. Wattanasakulpong, B. G. Prusty, D. W. Kelly and M. Hoffman, "A Theoretical Investigation on the Free Vibration of Functionally Graded Beams," Proceedings of the 10th International Conference on Computational Structures Technology, Valencia, 14-17 September 2010, p. 285.

[8] E. Alshorbagy Amal, M. A. Eltaher and F. F. Mahmoud, "Free Vibration Characteristics of a Functionally Graded Beam by Finite Element Method," Applied Mathematical Modelling, Vol. 35, No. 1, 2011, pp. 412-425. doi:10.1016/j.apm.2010.07.006

[9] J. Yang and Y. Chen, "Free Vibration and Buckling Analysis of Functionally Graded Beams with Edge Cracks," Composite Structures, Vol. 83, No. 1, 2008, pp. 4860. doi:10.1016/j.compstruct.2007.03.006

[10] S. Kitipornchai, L. L. Ke, J. Yang and Y. Xiang, "Nonlinear Vibration of Edge Cracked Functionally Graded Timoshenko Beams," Journal of Sound and Vibration, Vol. 324, No. 3-5, 2009, pp. 962-982. doi:10.1016/j.jsv.2009.02.023

[11] S. Sahraee and A. R. Saidi, "Free Vibration and Buckling Analysis of Functionally Graded Deep Beam-Columns on Two-Parameter Elastic Foundations Using the Differential Quadrature Method," Journal of Mechanical Engineering Science, Vol. 223, No. 6, 2009, pp. 1273-1284. doi:10.1243/09544062JMES1349

[12] S. C. Pradhan and T. Murmu, "Thermo-Mechanical Vi- 
bration of FGM Sandwich Beam under Variable Elastic Foundations Using Differential Quadrature Method," Journal of Sound and Vibration, Vol. 321, No. 1-2, 2009, pp. 342-362. doi:10.1016/j.jsv.2008.09.018

[13] J. C. Hsu, H. Y. Lai and C. K. Chen, "Free Vibration of Non-Uniform Euler-Bernoulli Beams with General Elastically End Constraints Using Adomain Modified Decomposition Method," Journal of Sound and Vibration, Vol. 318, No. 4-5, 2008, pp. 965-981.

[14] H. Y. Lai, J. C. Hsu and C. K. Chen, "An Innovative Eigenvalue Problem Solver for Free Vibration of EulerBernoulli Beam by Using the Adomain Decomposition Method," Computers \& Mathematics with Applications, Vol. 56, No. 12, 2008, pp. 3204-3220. doi:10.1016/j.camwa.2008.07.029

[15] Y. Liu and C. S. Gurram, "The Use of He's Variational Iteration Method for Obtaining the Free Vibration of an Euler-Bernoulli Beam," Mathematical and Computer Modelling, Vol. 50, No. 11-12, 2009, pp. 1545-1552. doi:10.1016/j.mcm.2009.09.005

[16] Q. Mao and S. Pietrzko, "Free Vibration Analysis of Stepped Beams by Using Adomian Decomposition Method," Applied Mathematics and Computation, Vol. 217, No. 7, 2010, pp. 3429-3441. doi:10.1016/j.amc.2010.09.010

[17] J. K. Zhou, "Differential Transformation and Its Application for Electrical Circuits," Huazhong University Press,
Wuhan, 1986.

[18] M. Malik and H. H. Dang, "Vibration Analysis of Continuous Systems by Differential Transformation," Applied Mathematics and Computation, Vol. 96, No. 1, 1998, pp. 17-26. doi:10.1016/S0096-3003(97)10076-5

[19] M. O. Kaya and O. O. Ozgumus, "Flexural-Torsional-Coupled Vibration Analysis of Axially Loaded Closed-Section Composite Timoshenko Beam by Using DTM," Journal of Sound and Vibration, Vol. 306, No. 3-5, 2007, pp. 495-506.

[20] O. O. Ozgumus and M. O. Kaya, "Flapwise Bending Vibration Analysis of Double Tapered Rotating Euler-Burnoulli Beam by Using the Differential Transform Method," Meccanica, Vol. 41, No. 6, 2006, pp. 661-670. doi:10.1007/s11012-006-9012-z

[21] O. O. Ozgumus and M. O. Kaya, "Vibration Analysis of a Rotating Tapered Timoshenko Beam Using DTM," Meccanica, Vol. 45, No. 1, 2010, pp. 33-42. doi:10.1007/s11012-009-9221-3

[22] S. C. Pradhan and G. K. Reddy, "Buckling Analysis of Single Walled Carbon Nanotube on Winkler Foundation Using Nonlocal Elasticity Theory and DTM," Computational Materials Science, Vol. 50, No. 3, 2011, pp. 10521056. doi:10.1016/j.commatsci.2010.11.001

[23] F. Delale and F. Erdogan, "The Crack Problem for a NonHomogeneous Plane," Journal of Applied Mechanics, Vol. 50, No. 3, 1983, pp. 609-614. doi:10.1115/1.3167098 


\section{Appendix A}

\section{S-S}

The frequency equation:

$$
\begin{aligned}
& \sum_{r=0}^{R} \frac{\omega^{2 r} I_{0}^{r} L^{(4 r+1)}}{\lambda^{r}(4 r+1) !} \times \sum_{r=0}^{R} \frac{\omega^{2 r} I_{0}^{r} L^{(4 r+1)}}{\lambda^{r}(4 r+1) !} \\
& -\sum_{r=1}^{R} \frac{\omega^{2 r} I_{0}^{r} L^{(4 r-1)}}{\lambda^{r}(4 r-1) !} \times \sum_{r=0}^{R} \frac{\omega^{2 r} I_{0}^{r} L^{(4 r+3)}}{\lambda^{r}(4 r+3) !}=0
\end{aligned}
$$

The mode shape function:

$$
\begin{aligned}
W(x)= & \sum_{r=0}^{R} \frac{\omega^{2 r} I_{0}^{r}}{\lambda^{r}(4 r+1) !} x^{(4 r+1)} \\
& -\frac{\sum_{r=0}^{R} \frac{\omega^{2 r} I_{0}^{r} L^{(4 r+1)}}{\lambda^{r}(4 r+1) !}}{\sum_{r=0}^{R} \frac{\omega^{2 r} I_{0}^{r} L^{(4 r+3)}}{\lambda^{r}(4 r+3) !}} \sum_{r=0}^{R} \frac{\omega^{2 r} I_{0}^{r}}{\lambda^{r}(4 r+3) !} x^{(4 r+3)}
\end{aligned}
$$

S-C

The frequency equation:

$$
\begin{aligned}
& \sum_{r=0}^{R} \frac{\omega^{2 r} I_{0}^{r} L^{(4 r+1)}}{\lambda^{r}(4 r+1) !} \times \sum_{r=0}^{R} \frac{\omega^{2 r} I_{0}^{r} L^{(4 r+2)}}{\lambda^{r}(4 r+2) !} \\
& -\sum_{r=0}^{R} \frac{\omega^{2 r} I_{0}^{r} L^{(4 r)}}{\lambda^{r}(4 r) !} \times \sum_{r=0}^{R} \frac{\omega^{2 r} I_{0}^{r} L^{(4 r+3)}}{\lambda^{r}(4 r+3) !}=0
\end{aligned}
$$

The mode shape function:

$$
\begin{aligned}
W(x)= & \sum_{r=0}^{R} \frac{\omega^{2 r} I_{0}^{r}}{\lambda^{r}(4 r+1) !} x^{(4 r+1)} \\
& -\frac{\sum_{r=0}^{R} \frac{\omega^{2 r} I_{0}^{r} L^{(4 r+1)}}{\lambda^{r}(4 r+1) !}}{\sum_{r=0}^{R} \frac{\omega^{2 r} I_{0}^{r} L^{(4 r+3)}}{\lambda^{r}(4 r+3) !}} \sum_{r=0}^{R} \frac{\omega^{2 r} I_{0}^{r}}{\lambda^{r}(4 r+3) !} x^{(4 r+3)}
\end{aligned}
$$

S-F

The frequency equation:

$$
\begin{aligned}
& \sum_{r=1}^{R} \frac{\omega^{2 r} I_{0}^{r} L^{(4 r-1)}}{\lambda^{r}(4 r-1) !} \times \sum_{r=0}^{R} \frac{\omega^{2 r} I_{0}^{r} L^{(4 r)}}{\lambda^{r}(4 r) !} \\
& -\sum_{r=1}^{R} \frac{\omega^{2 r} I_{0}^{r} L^{(4 r-2)}}{\lambda^{r}(4 r-2) !} \times \sum_{r=0}^{R} \frac{\omega^{2 r} I_{0}^{r} L^{(4 r+1)}}{\lambda^{r}(4 r+1) !}=0
\end{aligned}
$$

The mode shape function:

$$
\begin{aligned}
W(x)= & \sum_{r=0}^{R} \frac{\omega^{2 r} I_{0}^{r}}{\lambda^{r}(4 r+1) !} x^{(4 r+1)} \\
& -\frac{\sum_{r=1}^{R} \frac{\omega^{2 r} I_{0}^{r} L^{(4 r-1)}}{\lambda^{r}(4 r-1) !}}{\sum_{r=0}^{R} \frac{\omega^{2 r} I_{0}^{r} L^{(4 r+1)}}{\lambda^{r}(4 r+1) !}} \sum_{r=0}^{R} \frac{\omega^{2 r} I_{0}^{r}}{\lambda^{r}(4 r+3) !} x^{(4 r+3)}
\end{aligned}
$$

C-F

The frequency equation:

$$
\begin{aligned}
& \sum_{r=0}^{R} \frac{\omega^{2 r} I_{0}^{r} L^{(4 r)}}{\lambda^{r}(4 r) !} \times \sum_{r=0}^{R} \frac{\omega^{2 r} I_{0}^{r} L^{(4 r)}}{\lambda^{r}(4 r) !} \\
& -\sum_{r=1}^{R} \frac{\omega^{2 r} I_{0}^{r} L^{(4 r-1)}}{\lambda^{r}(4 r-1) !} \times \sum_{r=0}^{R} \frac{\omega^{2 r} I_{0}^{r} L^{(4 r+1)}}{\lambda^{r}(4 r+1) !}=0
\end{aligned}
$$

The mode shape function:

$$
\begin{aligned}
W(x)= & \sum_{r=0}^{R} \frac{\omega^{2 r} I_{0}^{r}}{\lambda^{r}(4 r+2) !} x^{(4 r+2)} \\
& -\frac{\sum_{r=0}^{R} \frac{\omega^{2 r} I_{0}^{r} L^{(4 r)}}{\lambda^{r}(4 r) !}}{\sum_{r=0}^{R} \frac{\omega^{2 r} I_{0}^{r} L^{(4 r+1)}}{\lambda^{r}(4 r+1) !}} \sum_{r=0}^{R} \frac{\omega^{2 r} I_{0}^{r}}{\lambda^{r}(4 r+3) !} x^{(4 r+3)}
\end{aligned}
$$

C-C

The frequency equation:

$$
\begin{aligned}
& \sum_{r=0}^{R} \frac{\omega^{2 r} I_{0}^{r} L^{(4 r+2)}}{\lambda^{r}(4 r+2) !} \times \sum_{r=0}^{R} \frac{\omega^{2 r} I_{0}^{r} L^{(4 r+2)}}{\lambda^{r}(4 r+2) !} \\
& -\sum_{r=0}^{R} \frac{\omega^{2 r} I_{0}^{r} L^{(4 r+1)}}{\lambda^{r}(4 r+1) !} \times \sum_{r=0}^{R} \frac{\omega^{2 r} I_{0}^{r} L^{(4 r+3)}}{\lambda^{r}(4 r+3) !}=0
\end{aligned}
$$

The mode shape function:

$$
\begin{aligned}
W(x)= & \sum_{r=0}^{R} \frac{\omega^{2 r} I_{0}^{r}}{\lambda^{r}(4 r+2) !} x^{(4 r+2)} \\
& -\frac{\sum_{r=0}^{R} \frac{\omega^{2 r} I_{0}^{r} L^{(4 r+2)}}{\lambda^{r}(4 r+2) !}}{\sum_{r=0}^{R} \frac{\omega^{2 r} I_{0}^{r} L^{(4 r+3)}}{\lambda^{r}(4 r+3) !} \frac{\omega^{2 r} I_{0}^{r}}{\lambda^{r}(4 r+3) !} x^{(4 r+3)}}
\end{aligned}
$$

